\title{
Comparative Evaluation of Bond Strength of Different Luting Cements for Cementation of Stainless Steel Bands on Primary Molar Crowns (Stainless Steel and Zirconia Crowns): An In Vitro Study
}

\author{
Ritesh Kalaskar ${ }^{1}$, Rajesh ljalkar ${ }^{2}$, Ashita Kalaskar ${ }^{3}$, Shruti Balasubramanian ${ }^{4}$
}

\begin{abstract}
Aim and background: Primary teeth are the valuable assets of a child. Apart from the obvious function of delivering esthetics, their most important function is space maintenance for the succeeding permanent teeth. At times, due to unavoidable pathological conditions, there is premature loss of primary teeth. Space maintainers can either be banded to the enamel surfaces of healthy adjacent teeth or to the surfaces of full coronal restorations, the most common ones being stainless steel crowns (SSCs) and zirconia crowns (ZCs). Due to esthetic demands, ZCs have started replacing SSCs and therefore, there needs to be data on the banding of space maintainers on ZCs and the bond strengths obtained on cementations. Thus, the present study planned to evaluate and compare the bond strengths obtained by the banding of stainless steel bands (SSBs) over SSCs and ZCs using type I glass ionomer cement (GIC) and self-adhesive resins.

Materials and methods: Sixty primary right mandibular molars were divided into four groups, group I, with cementation of SSB on SSC with type I GIC; group II, with cementation of SSB on SSC with self-adhesive resin cement; group III, with cementation of SSB on ZC with type I GIC; and group IV, with cementation of SSB on ZC with self-adhesive resin cement.

Results: The mean bond strength value of GIC as luting cements in group I is $1.13 \pm 0.075 \mathrm{MPa}$. The mean bond strength value of self-adhesive resin as luting cements in group II is $1.70 \pm 0.104 \mathrm{MPa}$. The mean bond strength value of GIC as luting cements in group III is $1.38 \pm 0.100 \mathrm{MPa}$. The mean bond strength value of self-adhesive resin as luting cements in group IV is $2.06 \pm 0.119 \mathrm{MPa}$.

Conclusion: The bond strength of self-adhesive resin was higher when SSB was cemented over SSC when compared with the bond strength of GIC when SSB was cemented over ZC.

Keywords: Bond strength, Glass ionomer cements, Self-adhesive resin cement, Stainless steel crown, Zirconia crown.

International Journal of Clinical Pediatric Dentistry (2021): 10.5005/jp-journals-10005-2003
\end{abstract}

\section{INTRODUCTION}

Primary teeth are the valuable assets of a child. In children, primary teeth play a vital role in mastication, phonetics, esthetics, and act as a space maintainer for permanent teeth. A healthy primary dentition preserves space for permanent teeth and maintains arch integrity. Diverse dietary patterns make children more susceptible to dental caries and results in extensive crown restorations and, at times, premature loss of primary teeth. ${ }^{1}$ The premature loss of primary teeth results in various discrepancies like unpleasant esthetics crowding of teeth, spacing of teeth bodily movement, alteration in the rhythm of permanent tooth eruption, difficulty in masticatory and phonetic function, midline discrepancies, deviated occlusion, arch length-tooth material discrepancies, impaction of permanent teeth, and disturbance in the occlusion of permanent dentition. ${ }^{2}$

The best, natural, and conservative way to avoid these problems is to preserve the primary teeth in the arch till their normal time of exfoliation is attained. ${ }^{3}$ Justifiably, it is rightly quoted that primary teeth serve as the best space maintainers for permanent dentition. However, if premature extraction or loss of a tooth is unavoidable the safest option to maintain arch space is by placing a space maintainer, either a fixed or a removable one. Fixed space maintainers are usually indicated to maintain the space created by unilateral/bilateral premature loss of primary teeth in either of the arches. Various appliances such as lingual arch, nance palatal arch,
1,2,4 Department of Pediatric and Preventive Dentistry, Government Dental College and Hospital, Medical College Premises, Medical Square, Nagpur, Maharashtra, India

${ }^{3}$ Department of Oral Medicine and Radiology, Government Dental College and Hospital, Medical College Premises, Medical Square, Nagpur, Maharashtra, India

Corresponding Author: Ritesh Kalaskar, Department of Pediatric and Preventive Dentistry, Government Dental College and Hospital, Medical College Premises, Medical Square, Nagpur, Maharashtra, India, e-mail: riteshpedo@gmail.com

How to cite this article: Kalaskar R, ljalkar R, Kalaskar A, et al. Comparative Evaluation of Bond Strength of Different Luting Cements for Cementation of Stainless Steel Bands on Primary Molar Crowns (Stainless Steel and Zirconia Crowns): An In Vitro Study. Int J Clin Pediatr Dent 2021;14(6):762-767.

Source of support: Nil

Conflict of interest: None

trans-palatal arch, band, and loop space maintainers are used for maintaining space. ${ }^{4}$ For the fabrication of these space maintainers, banding is required over the crown or tooth surfaces. However, there exist certain clinical situations wherein primary second molar have been endodontically treated which restored with full coronal

() The Author(s). 2021 Open Access This article is distributed under the terms of the Creative Commons Attribution 4.0 International License (https://creativecommons. org/licenses/by-nc/4.0/), which permits unrestricted use, distribution, and non-commercial reproduction in any medium, provided you give appropriate credit to the original author(s) and the source, provide a link to the Creative Commons license, and indicate if changes were made. The Creative Commons Public Domain Dedication waiver (http://creativecommons.org/publicdomain/zero/1.0/) applies to the data made available in this article, unless otherwise stated. 
restoration and primary first molar was lost prematurely which pose a challenge to the pediatric dentist.

When there is premature loss of the adjacent tooth, banding can be done on this stainless steel crown (SSC) so that it can be used as an abutment for maintaining space. The literature review documented the bond strength between stainless steel bands (SSBs) and SSCs. Bawazir et al..$^{5}$ compared the bond strengths between molar bands and SSCs wherein glass ionomer cement (GIC), resin-modified glass ionomer cements (RMGICs), and polycarboxylate cements (PXCs) were used as luting cement. Simultaneously, they also assessed the effect of sandblasting on the inner aspect of the molar bands on the mean bond strengths value and found that PXC showed the highest bond strength while RMGICs showed the lowest. Sandblasting the inner surface of bands enhanced the bond strength of different cements. ${ }^{5}$

With growing general awareness, many parents and even children as young as 3 years are becoming conscious of their appearance. Parents and patients prefer tooth-colored restoration. Especially, in the last decade, tooth-colored restoration like zirconia crowns ( $Z C s)$ received tremendous popularity due to its superior property over the SSCs. Increasing demand for esthetic restorations has resulted in increased use of all-ceramic restorations, such as zirconium. ${ }^{6}$ In such cases where endodontically treated teeth are restored with ZCs and an adjacent tooth is prematurely lost, planning of a space maintainer is a dilemma for a pediatric dentist.

Appropriate luting cement is required for the SSBs cementation to adhere to the tooth or crown surface with sufficient bonding strength. Various luting materials are available in the market for cementations of SSBs, but literature has a lack of evidence regarding bond strength between ZCs and SSBs using different luting cements. So, the present study was planned to evaluate the bond strength between SSBs and ZCs using different luting cements.

\section{Materials and Methods}

The estimated sample size was 60 reference models which were divided into 4 groups of 15 each group. In which group I and group II cementation of prefabricated SSB over SSCs with type I GIC and self-adhesive resin cement, respectively. In which group III and group IV cementation of prefabricated SSB over ZCs with type I GIC and self-adhesive resin cement, respectively. The sample size was determined by a power analysis based on mean and standard deviation values derived from a study conducted by Bawazir et al. ${ }^{5}$ by using Epi info online software.

We have included the mandibular right primary molars (85) of SSCs size $3\left(3 \mathrm{M}^{\mathrm{TM}}\right.$ ESPE ${ }^{\mathrm{TM}}$ Dental Products, USA) and ZCs size- $4 \mathrm{n}$ (Kidse-Crown, India). Similarly, prefabricated SSBs were selected and clinically adapted for best fit to the SSCs and ZCs. Manufacturer's details of all materials are mentioned in Table 1.

\section{Fabrication of Reference Model}

All ivory teeth were mounted on a self-cured acrylic resin (DPI-RR) block. Crown preparation was done on the embedded ivory tooth as per the standard guidelines of SSC and ZC preparation.

Stainless steel crown and ZC were cemented on the prepared ivory tooth using type I GIC and self-adhesive resin cement, respectively. Prefabricated SSBs were selected and clinically adapted for best fit to all crowns. Inverted " $U$ " shaped 19-gauge stainless steel orthodontic wire was soldered to the midbuccal and midlingual portions of the SSB.
Table 1: Materials used in this study

\begin{tabular}{ll}
\hline Materials & Manufacturing company \\
\hline 30 stainless steel & (Kids-Bands, Thickness-0.04 inch, and \\
band (Size-30+) & Width-0.150 inch) \\
30 stainless steel & (Kids-Bands, Thickness-0.04 inch, and \\
band (Size-32) & Width-0.150 inch) \\
Type I glass & GC FUJI Type I, GC CORPORATION 76-1 HASU- \\
ionomer cement & UNUMA-CHO, ITABASHI-KU, TOKYO, JAPAN \\
Self-adhesive resin & MEDICEPT UK LTD, 2nd Floor Hygeia House \\
cement & 66 College Road, Harrow, Middlesex, HA1 1BE, \\
& United Kingdom \\
30 stainless steel & 3M ${ }^{\text {TM }}$ ESPE ${ }^{\mathrm{TM}}$ Dental Products, \\
crowns & USA \\
30 zirconia crowns & Kids-e-Crown, Office Building Number \\
& B1-27A, Vivek Apartment Santacruz West \\
& Mumbai- 400,054, Maharashtra, India \\
\hline
\end{tabular}

The same procedure was performed for all the SSB in each group.

\section{Cementation of Stainless Steel Band over Stainless Steel Crown and Zirconia Crown}

Thereafter, the cement was mixed according to the manufacturer's instructions. It was loaded on the inner surface of SSB and the band was seated over the crown with hand pressure using an SSB pusher. The excess cement was removed from the occlusal and cervical margins of bands and from the surface of the crown with a dry cotton roll. Self-adhesive cement was light-cured for 20 seconds by using a light-curing unit (LED-D Woodpecker Curing Light). The cement was allowed to set. Thereafter, all reference models were stored in a constant temperature water bath at $37.0^{\circ} \mathrm{C}$ for 24 hours (Fig. 1).

\section{Laboratory Procedure to Determine the Bond Strength of Different Luting Cements}

To determine the force required to de-bond SSB from SSC and ZC, Universal Testing Machine ${ }^{6}$ (sunshine industrial 50 Kn) (Fig. 2) was used which runs at a crosshead speed of $1 \mathrm{~mm} /$ minute. The reference model was placed in the lower jaw whereas the J-shaped hook was attached to the upper jaw of the machine. This hook was then anchored to the U-shaped wire which was soldered to the SSB as stated earlier. The machine works based on the pull-out test in which the SSB is pulled out in the occlusal direction. The applied force should start from zero and gradually increased. The loading is continued until the cemented prefabricated SSB shows the first dislodgement toward the occlusal direction from SSC and ZC (Fig. 3 and 4). The machine is stopped by manual pressing of the button after the prefabricated SSB shows the first dislodgement and the readings are then recorded. The same procedure was performed for all the reference models in each group. The de-bonding force was recorded in Newton $(\mathrm{N})$.

\footnotetext{
Measuring bond strength of luting material in Mega Pascal (MPa)

Maximum force required separating the band from the crown $(\mathrm{N})$

Circumferential area of band $\left(\mathrm{mm}^{2}\right) \times$ Band Length $(L)(\mathrm{mm})$

Measuring of circumferential area of stainless steel band $\left(\mathrm{mm}^{2}\right)=\pi \times D$
} 


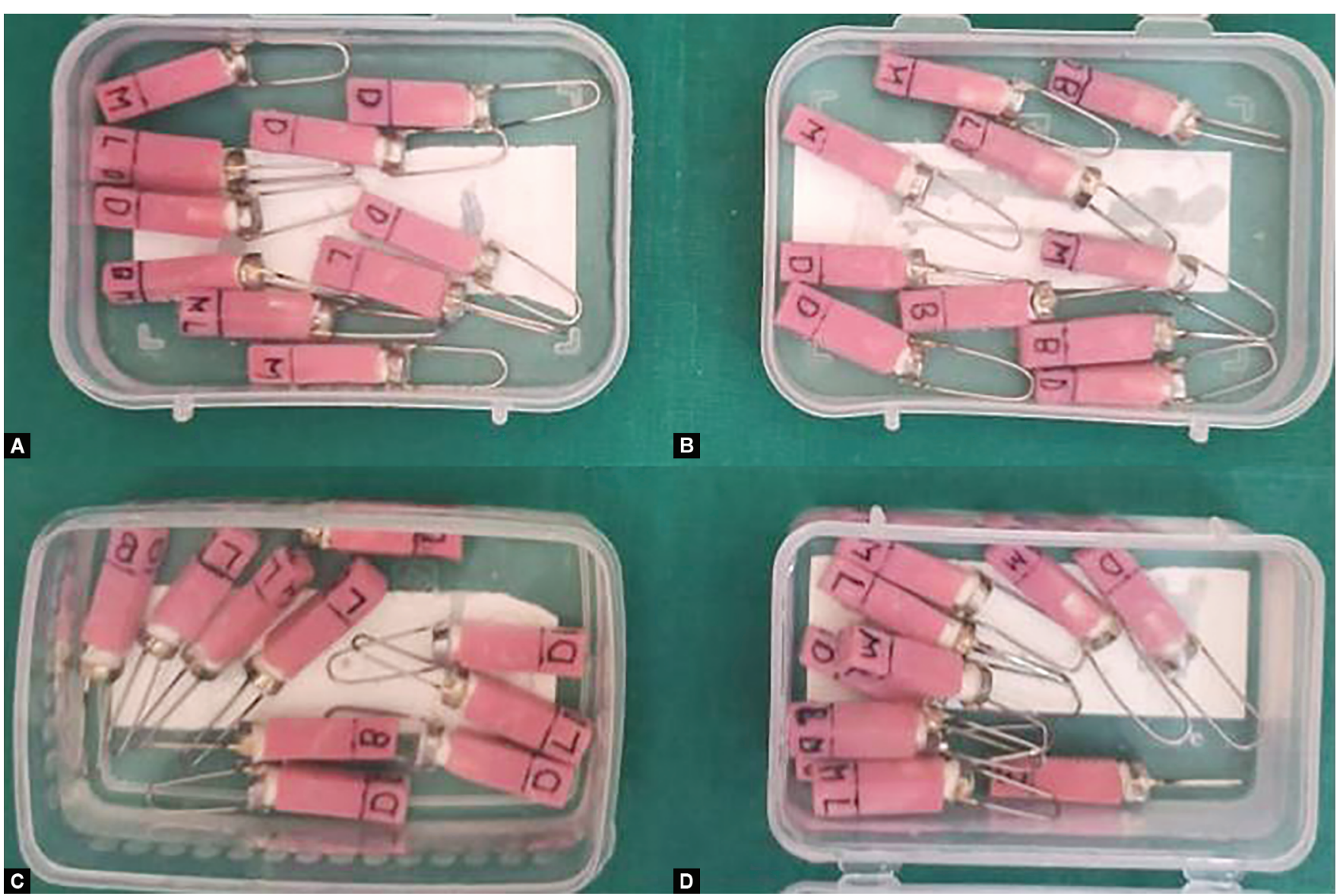

Figs $1 \mathrm{~A}$ to D: Reference model stored in a constant temperature water bath at $37^{\circ} \mathrm{C}$ for 24 hours

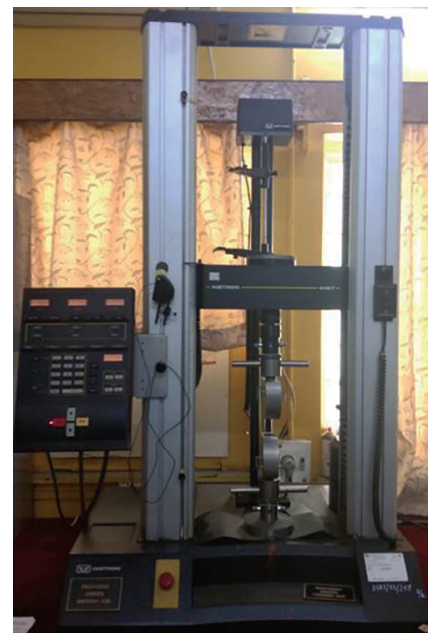

Fig. 2: Universal testing machine

\section{Statistical Analysis}

All the data obtained were compiled on an MS Office Excel Sheet (v 2019, Microsoft Redmond Campus, Redmond, Washington, United States). Data were subjected to statistical analysis using the Statistical Package for Social Sciences (SPSS v 26.0, IBM). Parametric tests have been used for comparisons. Intergroup comparison ( $>2$ groups) was done using one-way ANOVA followed by pair-wise comparison using post hoc test.

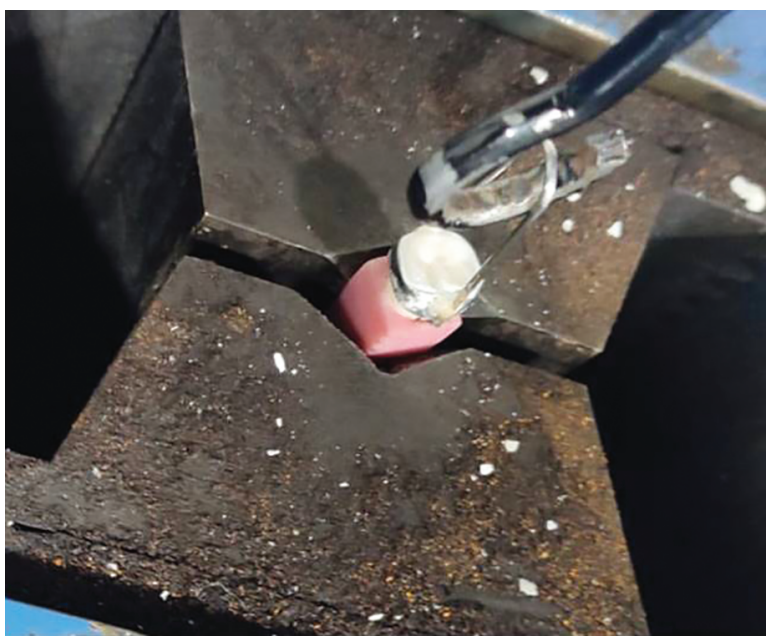

Fig. 3: Stainless steel band cemented over zirconia crown and mounted over universal testing machine

Results of the study were tabulated and evaluated using a one-way ANOVA test and a post hoc test for intergroup comparison. For all the statistical tests, $p<0.05$ was considered to be statistically significant.

$*$ Statistically significant difference $(p<0.05)$.

** = Statistically highly significant difference $(p<0.01)$.

$\#=$ Non-significant difference $(p>0.05)$ 


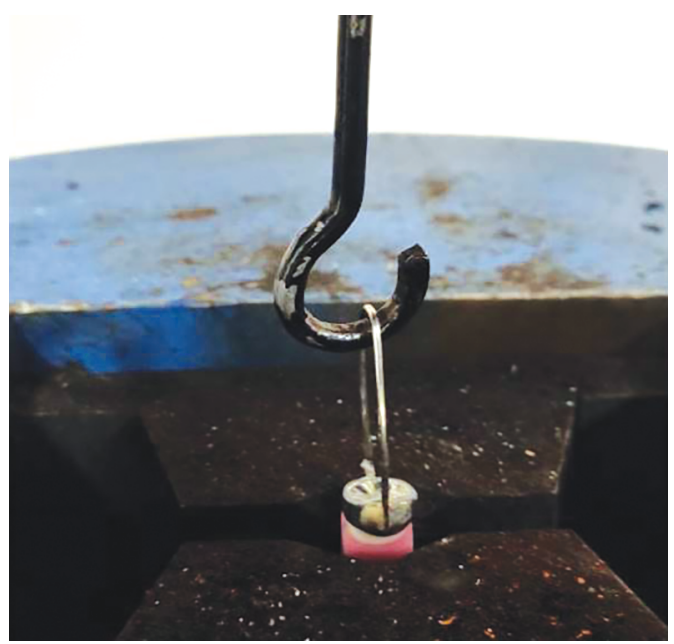

Fig. 4: Stainless steel band cemented over stainless steel crown and mounted over universal testing machine

\section{Results}

The present study was carried out to compare the bond strength of different luting cements used for cementation of SSB over the SSCs and ZCs.

The mean bond strength value of GIC as luting cements in group I is $1.13 \pm 0.075 \mathrm{MPa}$. The mean bond strength value of self-adhesive resin as luting cements in group II is $1.70 \pm 0.104 \mathrm{MPa}$. The mean bond strength value of GIC as luting cements in group III is $1.38 \pm$ $0.100 \mathrm{MPa}$. The mean bond strength value of self-adhesive resin as luting cements in group IV is $2.06 \pm 0.119 \mathrm{MPa}$.

The bond strength value was highest in group IV followed by group II, III, and I. One-way ANOVA test was applied for the comparison of the mean of each group and the difference was highly significant ( $p$ value $<0.000$ ) (Table 2 , and Fig. 5).

\section{Discussion}

Apart from the obvious function of delivering esthetics, primary teeth have been credited with vital and distinct other roles, the most important one being that of space maintenance for the succeeding permanent teeth. However, at times, due to unavoidable pathological conditions like dental caries, cracked tooth syndrome, or periodontal diseases, there is premature loss of primary teeth or they are extracted before their physiological age of exfoliation. The space left behind them, which should ideally be utilized by the succeeding permanent teeth, gets compromised by the shifting and drifting of the remaining teeth in the oral cavity. Thus, the concept of space maintainers becomes a crucial part of the practice of pediatric dentistry.

Space maintainers are indicated in clinical scenarios where there is premature loss of primary first molar. In situations like these, banding is done over the adjacent tooth to fabricate the space maintainer. However, there are instances wherein the adjacent tooth is already restored with SSC. In such conditions, banding of SSB over SSCs is advocated. The type of luting cement used for cementation of the SSB over the SSCs plays a crucial role in achieving optimum clinical results. Various luting cements have been used for the cementation of SSB over SSCs like GICs, self-adhesive resins, RMGICs, ZPC, PXCs, and resins. ${ }^{7}$

In the present study, we compared the bond strength of type I GIC and self-adhesive resin cement used for cementation of SSBS

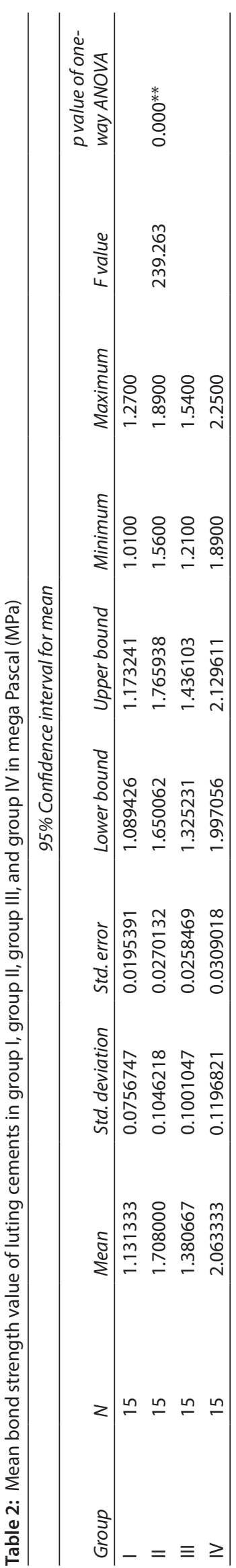




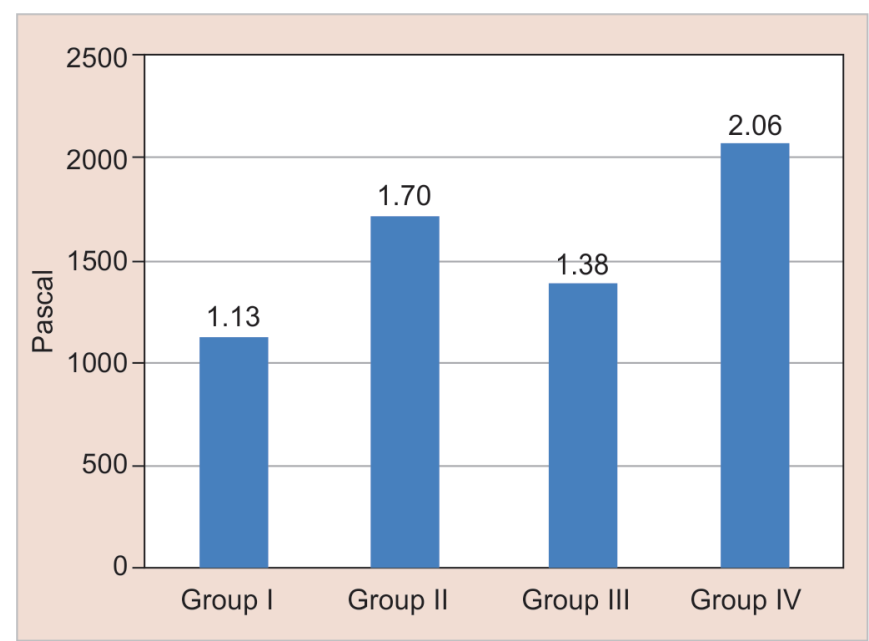

Fig. 5: Mean bond strength value of luting cements in group I, group II, group III, and group IV in mega Pascal (MPa)

over ZCs and SSCs. It was found that the best bond strength was acquired with self-adhesive resin cements on ZCs, followed by the same cement on SSCs and then the GICs on ZCs and SSCs. That means, the self-adhesive resin cements exhibited better bond strengths than GIC, irrespective of the material of the crown on which the SSBs were banded. This was in accordance with the study conducted by Srivastava et al. ${ }^{8}$ who compared the properties of both these cements with SSBs banded on extracted tooth surfaces. They suggested that self-adhesive resin cements were best luting cements in terms of their retentive properties and minimum microleakage when used for the cementation of SSBs.

We found that for the banding of SSBs on SSCs, self-adhesive resin cements were better than GIC, with a statistically significant difference in the bond strengths. For the successful banding on SSCs, various techniques have been followed in the past, including scoring of the bands with diamond burs, ${ }^{9}$ use of conventional and nanocomposites, ${ }^{10}$ employing surface conditioning treatments like aluminablasting, ${ }^{11}$ acid-etching, Nd:YAG laser surface treatment, ${ }^{12}$ and sandblasting, ${ }^{5}$ and the use of luting cements like resins, GICs, zinc phosphate, polycarboxylate, ${ }^{13}$ and self-adhesive resins. ${ }^{14}$ Our results were in accordance with the findings of Elsoud et al. ${ }^{14}$ who proposed that the self-adhesive resins had significantly higher retentive values than $\mathrm{RMGIC}$, that too, both in saliva-uncontaminated and saliva-contaminated surfaces of teeth. ${ }^{9}$

On comparing the type of crown on which the SSBs were banded, GIC showed better strength with SSBs banded on ZCs when compared with when banded on SSCs. Furthermore, it was observed in our study that the bond strength of self-adhesive resin was higher when SSB was cemented over ZC when compared with the bond strength of GIC when SSB was cemented over SSC. Özcan and Bernasconi ${ }^{15}$ in their systematic review analyzed the adhesion potential of resin-based and glass-ionomer luting cements to zirconia and highlighted the possible dominant factors affecting the bond strength results to this substrate. They concluded that an increased adhesion could be expected after physicochemical conditioning of zirconia and MDP-based resin cements presented higher results than those of other cement types when tested using macro- and microtensile tests.
Due to recent advancements in the field of ZCs and the short span of their implication in pediatric dentistry, substantial data on the banding of SSBs to ZCs are not available.

To the best of our knowledge, there are no studies reported in the literature that compare the bond strengths of SSBs when banded on SSCs and ZCs using GIC and self-adhesive resin cement. Only one study reported by Park et al. ${ }^{16}$ compared the bonding strengths on both these types of crowns, however, they used compomer and resin cements as luting cements. And they also found that the bond strength was maximum when resin cement was used on ZCs. Furthermore, they too found that bond strength was better when bands were looted on ZCs when compared with when luted on SSCs, irrespective of the luting cement used.

The limitations of our study included a matter of concern that we encountered during the conduction of our study, which was, that of the 15 samples of ZCs being banded with self-adhesive resin cement, 3 ZCs got dislodged from the teeth during band removal. This might be due to the excessive bond strength achieved between the SSBs and the ZCs due to the self-adhesive resin luting cements. Although we replaced the $\mathrm{ZCs}$ on the teeth to continue our study, we could not establish a justifiable answer to the occurrence. Also, such an occurrence has not been mentioned in any other relevant study. Thus, this occurrence must be considered and studied with a larger sample size in future studies. Furthermore, we suggest similar studies to be conducted in in vivo settings to consider the effects of saliva, gingival crevicular fluid, and masticatory forces on the bond strengths of SSBs on SSCs and ZCs.

Thus, through our study, we definitely give a better hope for the pediatricians and the parents of the patients who are concerned about the esthetics and therefore want to opt for ZCs and still get space maintainers designed for further esthetically improved results during the phase of permanent dentition. We suggest the use of self-adhesive resin cements for the banding of SSBs on ZCs, and encourage the use of ZCs and stainless-steel space maintainers, simultaneously, for the most effective esthetic results in a child.

\section{Conclusion}

In the present study, it was found that the best bond strength was acquired with self-adhesive resin cements on ZCs, followed by the same cement on SSCs and then the GICs on ZCs and SSCs. That means, the self-adhesive resin cements exhibited better bond strengths than $\mathrm{GIC}$, irrespective of the material of the crown on which the SSBs were banded.

It was evident in our study that the strengths rendered by the self-adhesive resins were higher when compared with similar groups employing the use of GICs, thus proving the superiority of self-adhesive resins as cementing agents over GIC.

\section{References}

1. Colak H, Dülgergil CT, Dalli M, et al. Early childhood caries update: a review of causes, diagnoses, and treatments. J Nat Sci Biol Med 2013;4(1):29-38. DOI: 10.4103/0976-9668.107257

2. Zou J, Meng M, Law CS, et al. Common dental diseases in children and malocclusion. Int J Oral Sci 2018;10(1):7. DOI: 10.1038/s41368-018-0012-3

3. Consolaro A. Should deciduous teeth be preserved in adult patients? How about stem cells? Is it reasonable to preserve them? Dental Press J Orthod 2016;21(2):15-27. DOI: 10.1590/21776709.21.2.015-027.oin

4. Setia V, Pandit IK, Srivastava N, et al. Space maintainers in dentistry: past to present. J Clin Diagn Res 2013;7(10):2402-2405. DOI: 10.7860/JCDR/2013/6604.3539 
5. Bawazir OA, Elaraby $\mathrm{A}$, Alshamrani $\mathrm{H}$, et al. Effect of sandblasting and type of cement on the bond strength of molar bands on stainless steel crowns. J Dent Child(Chic) 2015;82(2):64-69.

6. Raut $\mathrm{A}$, Rao PL, Ravindranath T. Zirconium for esthetic rehabilitation: an overview. Indian J Dent Res 2011;22(1):140-143. DOI: 10.4103/09709290.79979

7. Seale NS. The use of stainless steel crowns. Pediatr Dent 2002;24(5):501-505.

8. Srivastava N, Tyagi M, Rana V, et al. Comparative evaluation of different properties of various luting agents used for cementing stainless steel bands on molars. An ex-vivo study. EC Dent Sci 2019(9): 934-942.

9. Beemer RL, Ferracane JL, Howard HE. Orthodontic band retention on primary molar stainless steel crowns. Pediatr Dent 1993;15(6): 408-413.

10. Khatri A, Nandlal B, Srilatha. Comparative evaluation of shear bond strength of conventional composite resin and nanocomposite resin to sandblasted primary anterior stainless steel crown. $J$ Indian Soc Pedod Prev Dent 2007;25(2):82-85. DOI: 10.4103/09704388.33453
11. Stewart D, Tufekci E, Moon pc, Lindauer S. Bonding orthodontic brackets to stainless steel crowns. AADR annual meeting. 2010.

12. Grover N, Nandlal B. An in vitro evaluation of the effect of sandblasting and laser surface treatment on the shear bond strength of a composite resin to the facial surface of primary anterior stainless steel crowns. J Clin Exp Dent 2015;7(1):e119-e125. DOI: 10.4317/jced.51876

13. Parisay I, Khazaei Y. Evaluation of retentive strength of four luting cements with stainless steel crowns in primary molars: an in vitro study. Dent Res J (Isfahan) 2018;15(3):201-207. DOI: 10.4103/17353327.231864

14. Elsoud A, Hammouda H, Abdellatif A. Effect of teeth contamination on the retention of luted stainless steel crowns on primary molars. Acta Scienti Dent Sci 2009;3(5):39-44.

15. Özcan M, Bernasconi M. Adhesion to zirconia used for dental restorations: a systematic review and meta-analysis. J Adhes Dent 2015;17(1):7-26. DOI: 10.3290/j.jad.a33525

16. Park $\mathrm{C}$, Lee J, Lee $\mathrm{H}$, et al. Bond strength of band on zirconia crown with compomer and resin cement. J Korean Acad Pediatr Dent 2019;46(2):127-134. DOI: 10.5933/JKAPD.2019.46.2.127 